\title{
The effects of certain vegetable vs. particular meat breakfasts on the magnitude of human positive contrast and self-ratings of positive emotionality
}

\author{
LAWRENCE WEINSTEIN \\ Saint Leo College, Saint Leo, Florida 33574
}

\begin{abstract}
People who ingested certain vegetables for breakfast rated themselves more positively emotional than did people who ate particular meat breakfasts when daily body weights and caloric, water, and nutrient intakes were equated for the vegetable and meat groups. Furthermore, when an increase incentive magnitude produced positive contrast in the vegetable subjects and not in the meat eaters, the subjects who displayed the upward effect also rated themselves as happier than did the vegetable-unchanged control subjects. The present contrast data of shiftcontrol group appetitive emotionality differences and previous contrast results in the literature are in agreement with the notion that positive contrast is due to some appetitive emotional variable. The facts that (1) certain vegetable breakfasts produced differences in emotionality self-ratings and (2) particular vegetable breakfasts produced upward contrast are both consistent with some recent research findings of behavioral differences as a function of vegetable vs. meat regimes.
\end{abstract}

Little empirical information is known on the effects of vegetarian breakfasts vs. meat breakfasts when daily caloric intake, quality (type) of food, water intake, and body weights are equated on particular measures of human emotionality. A recent study has demonstrated that vegetable breakfasts resulted in higher university grades than meat breakfasts (Weinstein \& Staubi, 1977), but very few, if any, investigations have examined the pleasant emotionality effects of vegetable vs. meat breakfasts using the above controls. The present study examined the effects of vegetariansm vs. "meatariansm" at breakfast by employing the above control procedures on human subjects: (1) self-rating scale measures of positive emotionality and (2) measures of the magnitude of positive incentive contrast effects. The positive incentive contrast effect occurs when an increment in incentive magnitude results in a level of behavior that is significantly higher than the performance of a control group that has been continually exposed to the lower reward amount. The phemonenon is relatively well documented as being due to some emotional effect in humans and rats (e.g., Weinstein, 1971, 1972, respectively). According to such an emotional account of upward contrast, an increase in amount of reward results in an appetitive emotional response that produces internal cues that energize the instrumental response, thus resulting in an increase in performance and

Requests for reprints should be sent to Lawrence Weinstein, Department of Psychology, Saint Leo College, Saint Leo, Florida 33574. producing the upward effect. Further support for an emotional interpretation of upward contrast would be that on the upshifted sessions, subjects who experience an increase in incentive amount rate themselves more positively emotional (happier) on a self-rating scale than do control subjects maintained on the lower reward magnitude. Given that the positive contrast effect is due to some form of emotionality, one would expect that a group that was in some way more sensitive to the energizing effects of environmental change (i.e., more emotional) would display larger upward contrast effects than would another, less sensitive group. The present study examined (1) the self-rating scale measure of subjects who ate either vegetables or meat for breakfast when total daily caloric intakes, quality (types) of food, water intakes, and body weights were equated for the two groups and (2) the magnitude of positive incentive contrast effects as a function of vegetable or meat breakfasts, employing the above control procedures. If vegetables produce differences in experienced appetitive emotional responses, one would expect different rating scale measures for vegetable and meat eaters and different amounts of positive contrast for the two groups.

\section{METHOD}

Subjects

The subjects, 40 males enrolled at the University of British Columbia and the British Columbia Institute of Technology, were assigned randomly to two equal groups, vegetable breakfasts (V subjects) and meat breakfasts (M subjects). 


\section{Apparatus and Procedure}

For 2 initial adaptation months, the intended $V$ and $M$ groups' body weights were recorded. In addition, each group was instructed to eat the same total calories, 2,300, of the same types of foods (proteins, carbohydrates, and fats) for each day and for each meal. So $V$ subjects ate the same amounts of proteins, carbohydrates, and fats (measured in grams) as $\mathbf{M}$ subjects at each meal and over the entire day. Furthermore, each group was told to drink eight glasses of water per day and five cups of black coffee each day. The 2,300 calories were ingested as follows: 200 for breakfast (8:00 a.m.), 1,050 for lunch (12:00 noon), and 1,050 for supper (6:00 p.m.). On each day, at times randomly determined by the experimenter, each $\mathrm{V}$ and $\mathrm{M}$ subject was asked to rate himself on a scale from 0 ("Today, I do not feel good emotionally, or happy") to 20 ("Today, I feel very good emotionally, or happy"). Daily body weights were also recorded at the same times for $V$ and $M$ subjects.

For the next (breakfast-adaptation) month, $\mathrm{V}$ subjects ate $336 \mathrm{~g}$ of raw carrots ( 120 calories) with $280 \mathrm{~g}$ of raw cauliflower (80 calories), totaling 200 calories at the breakfast meal, while $M$ subjects were instructed to eat solely $112 \mathrm{~g}$ (weighed raw) of dry-fried round steak (200 calories) for the 8:00 a.m. meal. Body weights during the breakfast-adaptation month were recorded each day, as in the first 2 months, but rating scale measures were not taken again until the following (test) month. During the breakfast-adaptation month, daily and per-meal calories were equated for $\mathrm{V}$ and $\mathrm{M}$ subjects. Each group ate 2,300 calories per day $(200,1,050$, and 1,050$)$ at the same times as in the first 2 months. The 12:00 noon and 6:00 p.m. meals for $V$ and $M$ subjects, as before, consisted of the same types of foods ( $V$ subjects ate the same total amount of protein, of carbohydrate, and of fat as $M$ subjects; i.e., within each group, different amounts of proteins, carbohydrates, and fats were ingested at each meal and per day, but the same amount of protein, carbohydrate, and fat between groups at each meal and per day were eaten). Furthermore, each group drank eight glasses of water and five cups of black coffee per day, as in the first 2 months.

In the test month, exactly the same procedure was followed as in the breakfast-adaptation month, except that rating scale measures were again recorded in the same fashion as in the 2 initial adaptation months.

On the contrast day, following the test month, the details of the apparatus and procedure were essentially the same as those in Weinstein (1971) in that a verbal reaction time response was employed (each subject worked 30 mental multiplication problems, presented on slides, with $35 \mathrm{sec}$ between problems; one correct or incorrect answer or $60 \mathrm{sec}$ was allowed for each problem). On the 1 (and only) contrast day immediately following the 8:00 a.m. meal, the $\mathrm{V}$ and $\mathrm{M}$ groups were each randomly assigned to two equal groups, so 15 people appeared in each of four groups. In one situation $15 \mathrm{~V}$ subjects received 20 cents after Trials 2, 3, 4, 6, 11, 14, 15, 18, 23, 24, 25, 26, and 28 (Group V20). Another $15 \mathrm{~V}$ subjects received 2 cents through Trial 14, followed by an increase to 20 cents on Trial 15 and on the previously designated remaining trials (Group V2-20). In a third condition $15 \mathrm{M}$ subjects were given 2 cents relatively continuously (Group M20). The final situation involved $15 \mathrm{M}$ subjects' receiving 2 cents through Trial 14 , followed by an increment to 20 cents on Trial 15 and thereafter (Group M2-20). After each of the 30 consecutive problems, each subject in each $\mathrm{V}$ and $\mathrm{M}$ group was asked to rate how emotional he felt from 0 ("Now, I do not feel good emotionally, or happy") to 20 ("Now, I feel very good emotionally, or very happy"), and each $\mathrm{V}$ and $\mathrm{M}$ subject was weighed before the 8:00 a.m. starting time.

\section{RESULTS}

For the 2 initial adaptation months, mean body weights and rating scale measures each showed no significance for $\mathrm{V}$ vs. $\mathrm{M}$ subjects [for mean body weight, $\mathrm{F}(1,38)=1.62, \mathrm{p}>.05$; for the mean rating scale measure, $F(1,38)=1.56, p>.05]$.

For the next (breakfast-adaptation) month, body weight data between Groups $V$ and $M$ were not statistically significant by analysis of variance (ANOVA) $[\mathrm{F}(1,38)=2.62, \mathrm{p}>.05]$.

For the test month, body weights were again the same between Groups V and M by ANOVA $[F(1,38)=$ $2.63, \mathrm{p}>.05]$, but the rating scale measure was statistically significant $[F(1,38)=6.34, p<.05]$. The mean rating scale measure over the month for $\mathrm{V}$ subjects was 16.87 and for $M$ subjects, 9.14. The weekly means for the rating scale measure for $\mathrm{V}$ subjects were $18.5,16.9$, 16.3, and 17.0; those for $M$ subjects were 8.02, 9.0, 9.56, and 9.14.

For the contrast day, body weight data indicated no significant difference between any of the four groups $[\mathrm{F}(3,36)=2.10, \mathrm{p}>.05]$ before the first trial.

Figure 1 suggests that for Trials 1-14 M2-20 reacted slower than all other groups, V2-20 responded slower than V20, and M20 replied slower than V20.

The mean latency from Trials 1-14 differed significantly among the four groups by ANOVA $[F(3,36)=$ $3.58, \mathrm{p}<.05]$. By Newman-Keuls comparisons, the differences between $M 2-20$ and each other group, between V2-20 and V20, and between M20 and V20 were all statistically significant ( $\mathrm{ps}<.05$ ).

Figure 1 indicates that on Trial 16, the V2-20 subjects abruptly reduced their mean latency to a level below the control condition (positive incentive contrast effects), while the M2-20 subjects gradually lowered their mean latency scores, eventually about equaling their control condition (no positive contrast was displayed by the $\mathrm{M}$ group).

The mean latency from Trials 16-19 differed signifi-

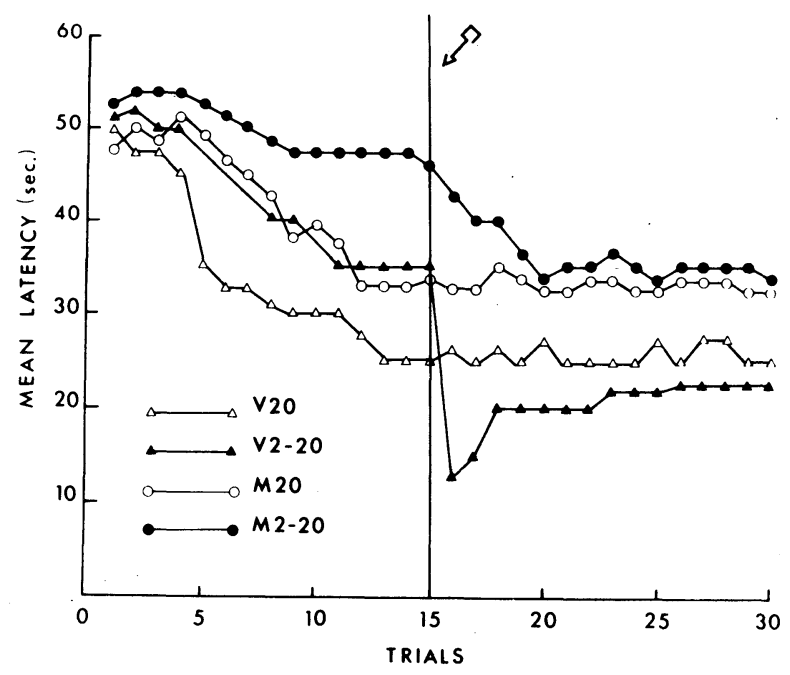

Figure 1. Mean latency in seconds. V2-20 and M2-20 shifted on Trial 15. Positive contrast was displayed over Trials 16-30 for $\mathrm{V}$ but not for $\mathrm{M}$ subjects. 
Table 1

Contrast Day

\begin{tabular}{lrrr}
\hline & \multicolumn{3}{c}{ Rating Scale Means Over Trials } \\
\cline { 2 - 4 } Group & $1-15$ & $16-19$ & $20-30$ \\
\hline V2-20 & 16.50 & 19.50 & 16.84 \\
V20 & 15.29 & 15.31 & 16.95 \\
M2-20 & 10.43 & 11.41 & 11.50 \\
M20 & 9.57 & 10.01 & 11.12 \\
\hline
\end{tabular}

cantly among the four groups by ANOVA $[F(3,36)=$ $5.62, \mathrm{p}<.01]$. By Newman-Keuls comparisons, the difference between V2-20 and V20 was significant $(\mathrm{p}<.01)$, but the difference between M2-20 and M20 was not ( $\mathrm{p}>.05)$.

The means for the emotional rating scale measure over the first 15 trials among the four groups on the contrast day was statistically significant by ANOVA $[F(3,36)=3.01, p<.05]$. By Newman-Keuls comparisons, the differences between V2-20 and M2-20 and between V20 and M20 were statistically reliable (ps <.05), but the differences between V2-20 and V20 and between M2-20 and M20 were not (ps>.05). For Trials 16-19 (the days Group V showed contrast), the rating scale means were statistically significant among the four groups by ANOVA $[F(3,36)=3.54$, $\mathrm{p}<.05]$. By Newman-Keuls comparisons, the difference between V2-20 and V20 was significant $(p<.05)$, but the difference between M2-20 and M20 was not ( $p>.05$ ). A final ANOVA on the rating scale measures among the four groups from Trial 20 to Trial 30 revealed a significant $F$ value $[F(3,36)=3.04, p<.05]$. By Newman-Keuls comparisons, the differences between V2-20 and M2-20 and between V20 and M20 were significant (ps $<.05)$, but the differences between V2-20 and V20 and between M2-20 and M20 were not ( $\mathrm{ps}>.05)$. The means are given in Table 1 .

\section{DISCUSSION}

Body weights never statistically varied between any of the groups throughout the study. In the test month the rating scale finding that Group V was significantly higher than Group M demonstrates that the particular vegetable breakfasts used herein resulted in subjects' reporting that they were more positively emotional compared with other subjects who ate the meat breakfasts used herein. It is also noted that during the initial 2 adaptation months, the $\mathrm{V}$ and $\mathrm{M}$ subjects rated themselves as no different in terms of emotionality. On the contrast day, the preshift results were similar to the findings in most incentive contrast studies (e.g., Weinstein, 1972). In other words, two discriminably different reward levels were used for $\mathrm{V}$ and $\mathrm{M}$ subjects, where discrepant amounts of reward are defined by the significantly different levels of performance they produce, rather than by different values on the stimulus scale (V20 vs. V2-20 and M20 vs. M2-20).
The preshift findings further indicated that the vegetable breakfasts used in this study produced faster responses in humans than did the particular meat breakfasts in this experiment for each reward level. So, V20 was quicker to react than $\mathrm{M} 20$, and V2-20 was faster to respond than M2-20. These data demonstrate the differential effect of certain vegetable breakfasts and certain meat breakfasts on latency of responding. The present findings that from Trial 16 to Trial 10 positive contrast occurred in V2-20 and that V2-20 subjects rated themselves as happier or feeling better than V20 subjects only when displaying contrast support the notion that upward incentive contrast effects are due to some form of appetitive emotionality. When positive contrast failed to occur on Trials 16-19, M2-20 subjects rated themselves as emotional as M20 subjects. As discussed earlier, the present findings of more pleasant emotionality in the upshifted V subjects than in the control V subjects supports earlier research attributing contrast to the energizing effects of a motivational variable (e.g., Crespi, 1942; Weinstein, 1972).

The present finding that a relatively well documented emotional effect (e.g., Weinstein, 1971, 1972), positive contrast, occurred only in $V$ subjects and not in $M$ subjects is not at variance with the notion that at least some vegetable breakfasts produce more appetitive emotionality in people than do certain meat breakfasts. The higher level of emotionality in Group V than in Group M on the contrast day (and previously throughout most of the study) would then quite possibly make the $\mathrm{V}$ subjects more prone to display an effect likely to be emotionally mediated, that is, positive incentive contrast.

The present findings of particular vegetable breakfasts' producing higher levels of rated positive emotionality (in the test month, for instance) than certain meat breakfasts and that vegetable ingestion resulted in positive contrast while meat intake did not produce the upward effect agree with recent research (e.g., Weinstein \& Staubi, 1977) in finding differences in felt frustration or emotional upset under vegetable vs. meat regimes (V subjects felt less frustrated than $M$ subjects, as measured by the Rosenzweig, 1967, Picture Frustration Test and a rating scale). Investigations are presently planned to examine vegetable-meat differences in behavior in terms of the permanence of such performance disparities. For example, how long do vegetarian/meatarian-mediated differences in felt emotionality prevail-for months, years?

\section{REFERENCES}

Crespi, L. Quantitative variations of incentive and performance in the white rat. American Journal of Psychology, 1942, 55, 467-517.

RoSENZWEIG, S. Revised criteria for the group conformity rating of the Rosenzweig Picture Frustration Test, adult form. Journal of Projective Techniques and Personality Assessment, 1967, 31, 58-61.

WEINSTEIN, L. Effects of an increment in monetary magnitude on instrumental responding and repeated increases in reward magnitude in humans. Psychonomic Science, 1971, 25, 235-237.

Weinstein, L. Contrast effects in animal and human learning: Recent results and interpretations. Journal of Psychology, 1972, 81, 235-247.

Weinstein, L., \& Staubi, W. H. W. Vegetables make you more successful at university than meat. International Research Communication Systems, 1977, 5, 44.

(Received for publication December 15, 1979.) 